\title{
Review of Prevention of Mother-To-Child Transmission (PMTCT) program in a Primary Healthcare Centre in Abuja, Nigeria
}

\author{
Article by Orji Ikechukwu Anthony ${ }^{1},{ }^{2}$ Okoli Ugochukwu Thompson \\ ${ }^{1}$ Disease Control Unit, Health Department, AMAC, Abuja, Nigeria \\ ${ }^{2}$ Clinical Services Department, Lighthouse Medicare Services Ltd. Abuja, Nigeria \\ E-mail: ${ }^{1}$ drtony2013@gmail.com, ${ }^{2}$ drtonyiyke@gmail.com
}

\begin{abstract}
Background: It has been shown that nearly all pediatric seropositive HIV cases were infected via their seropositive mothers, as well; almost all of these cases can be prevented through a robust PMTCT service that provides extremely effective Anti-retroviral therapy and prophylactic intervention (FMOH, 2010).

As we work towards elimination of MTCT of HIV in our country, the importance of this review of PMTCT services cannot be over emphasized.

Objectives: The objectives of the study were; to assess the prevalence of HIV among pregnant women who attended ANC at the Health center (January-December, 2015). 2. To evaluate the percentage of HIV positive pregnant women who were placed on ART in the review period. 3. To ascertain the percentage of babies born to HIV positive mothers who were given $A R T$ in the review period.

Methods: Secondary data review was employed in this descriptive cross-sectional study. It involved data extraction from ANC booking registers, PMTCT HIV screening registers, ART administration registers and case/delivery notes of HIV positive mothers, covering the review period of January-December 2015, in the study center.

Results: All the 1,418 ANC clients reviewed were screened for HIV with a sero-prevalence of $2.8 \%$. However, the percentage of seropositive clients who were placed on ART was $75 \%$, while, $25 \%$ were lost to follow-up. Furthermore, only $33 \%$ of the babies born to seropositive mothers received ART prophylaxis in this center.

Conclusion: An ANC sero-prevalence of $2.8 \%$ with below average ART initiation for positive pregnant women and babies born to them portends great danger towards elimination of MTCT of HIV. Therefore the study recommends urgent strengthening of PMTCT in this center and other similar centers across the country.
\end{abstract}

Keywords: PMTCT, Ante-Natal Care, HIV, Prevalence, Anti-Retroviral Therapy, Primary Health Centre

\section{Acronyms}

AIDS: Acquired Immune Deficiency Syndrome

ANC: Ante Natal Care

ART: Anti-Retroviral Therapy

ARV: Anti-Retroviral drugs

CDC: Center for Disease Control

FMOH: Federal Ministry of Health

GAC: Ghana AIDS commission

HAART: Highly Active Anti-Retroviral Therapy

HIV: Human Immunodeficiency Virus

HSS: Health Sector Strengthening

IATT: Inter-Agency Task Team

LSHTM: London School of Hygiene \& Tropical Medicine

MTCT: Mother-To-Child Transmission

NARHS: National HIV/AIDS and Reproductive Health Survey 
Texila International Journal of Public Health

Volume 4, Issue 4, Dec 2016

NACA: National Agency for Control of AIDS

PHC: Primary Healthcare Center

PLWHA: Persons Living with HIV \& AIDS

PMTCT: Prevention of Mother to Child Transmission

SDG: Sustainable Development Goals

UNICEF: United Nations Children's Fund

\section{Introduction}

Human Immunodeficiency Virus (HIV) is the virus that causes AIDS. They are of two types (HIV 1 \& HIV 2) and are of the retrovirus family (LSHTM, 2014). HIV is transmitted via direct contact with body fluids infected with HIV, like semen, blood and genital secretions; it can also be by vertical transmission from an HIV-infected mother to her child during pregnancy, delivery, or through breast milk during breastfeeding. The majority of the infection globally is caused by HIV-1 (AIDSINFO, 2016). Acquired Immune Deficiency Syndrome (AIDS) is a term which refers to the most advanced stages of the disease resulting from HIV infection. It is defined by the presence of any opportunistic infections (more than 20 of them) or HIV-related cancers $\left(\mathrm{WHO}_{1}, 2016\right)$. AIDS is a disease of the immune system resulting from HIV infection. HIV destroys the CD4 T lymphocytes (CD4 cells) of the immune system, thereby, leaving the body vulnerable to a number of life-threatening infections and cancers. To make the diagnosis of AIDS, the patient infected with HIV must have an AIDS-defining condition or have a CD4 count less than 200 cells $/ \mathrm{mm}^{3}$ (AIDSINFO, 2016)

According to the Nigeria's National PMTCT 2010 guideline, 370,000 children were newly infected with HIV in 2009 with $90 \%$ of these cases found in sub-Saharan Africa. Note that nearly all such infections can be prevented by a PMTCT program that provides highly effective ART and ARV prophylactic interventions. This follows that an HIV free generation cannot be realized without an absolutely effective PMTCT program. Nigeria currently harbors the $2^{\text {nd }}$ highest Global HIV burden, has a general population prevalence of $3.4 \%$ by NARHS survey report, (NACA 2014) and ANC prevalence of 3.0\% (FMOH, HSS, 2014). With the above staggering statistics, the importance of a robust PMTCT program in the effort to attain zero new infection cannot be over emphasized. This underscores the need at this period for a study on this subject, to begin assessing the current prevalence of HIV infection among pregnant mothers as well as evaluating the PMTCT services for the positive pregnant mothers and babies born to them. This is in line with achieving the sustainable development goal-3 on Good health and well-being which has one of its key targets as ending the epidemics of AIDS by 2030 (SDG, 2015). Universal access to HIV/AIDS care is crucial to ending the epidemic, thus, strengthening HIV care especially PMTCT services at Primary Health Centers is a vital to achieving this feat.

This study evaluated the PMTCT services at this center with a view to finding the gaps and making recommendation for improved service delivery.

\section{Objectives}

1. To assess the prevalence of HIV among pregnant women who attended ANC at the Health center (Jan. -Dec., 2015).

2. To evaluate the percentage of HIV positive pregnant women who were placed on ART in the review period according to National guideline.

3. To ascertain the percentage of babies born to HIV positive mothers who were given ART in the review period according to National guideline.

\section{General considerations}

HIV Infection: Since 1981, when the first case of HIV/AIDS infection was recorded in USA (LSHTM, 2014), the epidemic has been on the increase globally, especially affecting sub-Saharan Africa worse than any other region of the world. According to the 2014 NARHS 
report, Nigeria with $3.4 \%$ prevalence in the general population is now the $2^{\text {nd }}$ highest HIV burdened country behind only South Africa. Furthermore, the FMOH 2014 National HIV sero-prevalence sentinel survey among pregnant women attending ANC in Nigeria revealed a $3.0 \%$ prevalence rate accentuating the need for an effective PMTCT program to halt vertical transmission of HIV to infants.

PMTCT: Prevention of Mother to Child Transmission (PMTCT) of HIV is the transmission of HIV infection from a HIV Positive mother to her baby during pregnancy, delivery and breastfeeding $\left(\mathrm{WHO}_{2}, 2016\right)$. Between $15 \%-45 \%$ of these babies will be infected without any intervention, however, with effective PMTCT, infection rate can be reduced to just above $1 \%\left(\mathrm{WHO}_{2}, 2016\right)$. PMTCT involves treatment of the positive mother with highly active anti-retroviral drugs (HAART) and giving the baby a short course of antiretroviral drugs prophylactically. Moreover, measures to prevent HIV acquisition in the pregnant woman and appropriate breastfeeding practices are also included. Evidence of the efficacy of an effective PMTCT program in eliminating the vertical transmission of HIV from an infected mother to her baby is made bare in the recent validation of Thailand by World Health Organization, in June 2016, for eliminating mother to child transmission of HIV and Syphilis $\left(\mathrm{WHO}_{3}, 2016\right)$. This is the first in South East Asia as well as the first high HIV burdened country to have achieved such a milestone, hence, proving to the rest of the world that an HIV free generation is possible globally. It only requires sound HIV prevention program especially PMTCT and political will from the Government. According to World Health Organization's report, other countries who have reported similar validation for elimination of Mother to child transmission of diseases includes: Belarus (HIV\& Syphilis), Armenia (HIV), Republic of Moldova (Syphilis), $\left(\mathrm{WHO}_{4} 2016\right)$.

World Health Organization's guideline for the use of antiretroviral drugs for PMTCT: The World Health Organization $\left(\mathrm{WHO}_{5}\right)$ in the month of June, 2013, released the Consolidated Guidelines on the Use of Antiretroviral Drugs for Treating and Preventing HIV infection. This particular guidelines approved the initiation of Anti-Retroviral Therapy for all pregnant and breastfeeding women living with HIV. Lifelong Anti-Retroviral Therapy for this population is highly recommended, especially in generalized epidemics; irrespective of CD4 cell count (this is known as Option $\mathbf{B}+$ ) for both programmatic and operational reasons. Moreover, Countries may also choose to stop ART once risk of mother-to-child transmission has passed (i.e. at the end of breastfeeding or after delivery if no breastfeeding); unless the woman is eligible for lifelong Anti-Retroviral Therapy based on CD4 criteria (this is known as Option B). Note that in either setting, the woman continues with routine HIV care. The above 2013 guideline is still subsisting in many developing countries (due to resource limitations) even though a revised 2015 guideline has been issued by WHO which recommends option $\mathrm{B}+$ as the standard, thus, stating that ART should be initiated in all pregnant and breastfeeding women living with HIV, regardless of WHO clinical stage and at any CD4 cell count and continued lifelong $\left(\mathrm{WHO}_{5}, 2016\right)$.

Overview of HIV infection in Nigeria in relation to some African countries: According to Sagay et. al 2005, the prevalence of HIV among pregnant women was 8.2\% in Jos Nigeria in 2005. In this study, it was also found that Women aged 20-29 years had more than 4-fold increased risk of HIV. Moreover, women with multiple marriages and women married to husbands who had multiple partners were also found to have increased risk of HIV infection. In Ghana, the Ghana AIDS Commission (GAC) reports that 2013 HIV prevalence among pregnant women attending Antenatal clinic is 1.9\%, which is a drop from the 2012 prevalence of 2.1\% (GAC, 2013). However, this prevalence is higher than the National Prevalence of $1.3 \%$, thus, suggesting that more effort is needed to strengthen PMTCT in Ghana, if the dream of an AIDS free generation will be realized. Nevertheless, this is a far better statistics compared to Nigeria with general population prevalence of $3.4 \%$ (NARHS, NACA 2012) and ANC prevalence of 3.0\%. (FMOH, HSS, 2014), though, unlike the Ghana situation, the Prevalence of HIV infection among pregnant women is lower than the National general population prevalence. Okerentugba et. al (2015) in their study among pregnant women in a 
cottage hospital in Port Harcourt, Nigeria found a prevalence of 3.0\% same as the National prevalence according to FMOH/HSS (2014). Kiptoo et. al (2009) study in Kenya revealed an ANC prevalence of $6.7 \%$ higher than the Kenya national survey of same year which recorded 6.4\%, and 5.6\% by 2012 (Kenya AIDS response report, 2014). This is akin to the situation in Ghana where the ANC prevalence is higher than the National prevalence; therefore, more effort should be put into PMTCT to ensure transmission of HIV to children is eliminated. This is very fundamental bearing in mind that over $90 \%$ of HIV infection is via Mother to child transmission during pregnancy/delivery /breastfeeding.

Towards zero new HIV infection in Nigeria, the Place of PMTCT: To discuss the strategy of zero new infection in Nigeria, Thailand experience comes handy. According to Thailand National AIDS Committee (2015) as documented in the 2015 Thailand AIDS response progress report, Thailand is one of the countries in ASIA that subscribed to the UN General Assembly Special Session on HIV with a commitment to prevent and control the AIDS epidemic and pursue the strategy of three Zeroes: these are (i) Zero new HIV infection; (ii) Zero AIDS death; and (iii) Zero AIDS stigma and discrimination. The Country developed strategies to achieve the above, covering all aspects of HIV prevention, however, with regards to PMTCT specifically; the Thailand National strategic plan was to reduce peri-natal transmission of HIV to prevalence of less than $2.0 \%$ by 2016 as against 2014 prevalence of $2.1 \%$. The report further stated that Thailand has developed key measures and the operational plan for 2015-19 to support the policy of ending AIDS by the year 2030. The program applied the strategies of test and treat regardless of CD4 level with focus to the most affected areas and population including the PMTCT program. This has already yielded positive result as validated by World Health Organization, in June 2016 that Thailand has succeeded in eliminating Mother to child of HIV. Moreover, Thailand was one of the first countries in the Asia and Pacific region adopting the ambitious targets of providing anti-retroviral treatment to all people with HIV irrespective of CD4 count. The strategy also focused on early detection and treatment for key populations as well as improving access to services for non-Thais. This can be achieved in Nigeria by developing the right strategy to holistically tackle HIV prevention, especially paying adequate attention to PMTCT, and other forms of prevention. Making drugs available for all eligible patients for treatment, scaling up counseling and testing as well as removing all forms of discrimination and stigmatization of Persons Living with HID \& AIDS.

\section{Methods}

This is a retrospective cohort study that used the existing data for all pregnant women who attended ANC at the health center during the study review period (January -December, 2015). Data were extracted from PMTCT, ART and Antenatal registers as well as Delivery case notes of all the sero-positive clients. The study was carried out at Kagini Health Center, Gwagwa Ward, AMAC, Abuja. The site was purposively selected as it is a Primary Health Center within the ward with capacity to run a PMTCT service and has been offering the service for more than two years now. The Study Population involves a cohort of all pregnant women who attended ANC at the center from January to December, 2015. The Sample were selected using the convenient sampling method as it involved the review of all ANC records at the research site from January to December, 2015. The sample size was 1,418 pregnant women who attended ANC at the center during the review period. Data extraction strategy involves the following: 1 . The ANC booking register was reviewed, to determine the total number of pregnant women who were booked in this Health facility from January $1^{\text {st }}, 2015$ to December 31st, 2015. The booking practice is that HIV screening and counseling will be done free of charge on the first day of booking. 2. The PMTCT register was used to extract the results of the HIV screening done and the age range of the patients. 3. ART registers were reviewed to ascertain if HAART were given to the HIV positive pregnant women during the course of the ANC. 4. The case notes and the delivery files of the patients whose results were positive were reviewed to find out if ARV were given to the babies born to HIV positive 
mothers. The extracted data was analyzed by univariate analysis to make frequency tables and presented with pie, column and bar charts. Ethical approval was given by AMAC Health Department.

\section{Results}

1. The study found that 1,418 pregnant women were registered for ANC at the Kagini Health Center from January $1^{\text {st }}$ to December $31^{\text {st }}$, 2015. Moreover, $100 \%$ of these clients were counselled and screened for HIV 1 \& 2 antibody in accordance with the National guideline on PMTCT.

Age distribution of the clients were as follows: 15-24yrs (38\%), 25 - 34ys (50\%), 35yrs and above (12\%).

2. The sero-prevalence for HIV among pregnant women who attended ANC clinic at Kagini Health center in the year 2015 was 2.8\% (40/1418).

Age distribution of the positive clients were as follows: 15-24yrs (25\%), 25 - 34ys (60\%), $35 y$ rs and above (15\%).

3. The study also revealed that $75 \%$ (30/40) of the sero-positive pregnant patients received ARV therapy at this Health center. The remaining 25\% were lost to followup.

4. Furthermore, only 33.3\% (10/30) of babies born to these sero-positive mothers received ARV therapy at this Health center, $66.6 \%$ were lost to follow-up.

5. Concerning the point of HIV testing \& Counselling, $99.7 \%$ of the clients were screened at the ANC routine clinic while $0.3 \%$ were screened during labour as they were un-booked who did not attend ANC clinic.

6. Among the sero-positive clients who received ARV therapy, $30 \%$ were initiated at a Gestational age of 14 weeks -27weeks, while $70 \%$ were initiated at Gestational age of 28weeks -33weeks.

7. Additionally, $80 \%$ of the clients on ARV therapy were on triple regimen prophylaxis for PMTCT, while $20 \%$ were on lifelong ART.

\section{Discussions}

1. This center exhibited good practice with respect to testing all ANC clients for HIV $1 \& 2$ which is in line with the National PMTCT guideline. This will facilitate the diagnosis of new HIV infections as was the case in this center where 85\% (34/40) of all diagnosed cases (figure 7) and 80\% (24/30) of all cases on treatment (figure 6) were newly diagnosed cases. Detecting new infections and initiating them on ARV therapy is one sure way of ending this AIDS epidemic, therefore, this excellent practice should be encouraged and sustained.

2. The ANC sero-prevalence of $2.8 \%$ (figure 1 ) is similar to the National ANC seroprevalence of 3.0\%, (FMOH, HSS, 2014), which is still high, necessitating more effort to improve PMTCT services towards eliminating MTCT of HIV/AIDS in the near future. Furthermore, the age distribution of the positive clients revealed a high prevalence (50\%) for age group 25 - 34 years age group and 38\% for age group 15 24 which is similar to the findings of Sagay et. al (2009) where pregnant women aged 20-29 years had more than 4-fold increased risk of HIV infection.

3. $75 \%$ of the sero-positive pregnant patients received ARV therapy at this Health center while $25 \%$ of the clients were lost to follow-up (Figure 2). This finding is not a good practice and portends danger to the process of eliminating mother to child transmission of HIV. It was discovered that those patients who dropped out after their HIV positive status were diagnosed never received any followed-up visit/call because there was no provision made for such follow-up. There was neither any staff responsible nor any fund earmarked for the follow-up activities. This group of patients may end-up not taking the ARVs, thereby, increasing the risk of their babies getting HIV infection. This is an identified gap which as a matter of urgency must be 
addressed to strengthen the PMTCT program in this center in particular and other similar centers across the country. Further research in the area of finding reasons for drop-out may be able to proffer solutions to this challenge.

4. There is also a very poor performance indicator observed as regards the poor rate of initiation of ARV therapy and retention for the babies born to HIV positive mothers in this center. A 33.3\% rate (Figure 3) is abysmally poor against the expected $100 \%$ initiation of ARV therapy.

This also bothers on follow-up as applied to the initiation of ARV therapy to sero-positive pregnant clients, thus, similar solution applies.

5. The high percentage of clients tested during ANC visit which is $99.7 \%$ (Figure 4) depicts high awareness of the importance of ANC clinic within the community and its environ, thus, almost all the pregnant women accessing service at this facility attended ANC clinic. Only 0.3\% (4/1418) did not attend ANC but presented in labour and delivered at the Health center. This high ANC awareness is a strength that can be leveraged upon to eliminate MTCT of HIV in this Health Center.

6. Most of the sero-positive clients were initiated on ARV therapy by 28-33 weeks (70\%), while only $30 \%$ were initiated by $14-27$ weeks (Figure 5 ) which suggest late ANC booking by most of the clients. This is an area the Health workers at this center must work on, to enhance early ANC booking, early ARV initiation which will definitely improve the prevention of transmission of HIV from the sero-positive mother to their unborn children.

7. The study revealed that all the previously diagnosed pregnant patients adhered to taking ART during the course of their pregnancy 6/40 (20\%) of the diagnosed cases and 6/30 (15\%) of those who complied with ART treatment (Figures 6 and 7). Therefore, all the patients lost to follow-up were newly diagnosed sero-positive patients. This suggest, probably poor counselling activities (post-test counselling, preinitiation counselling, adherence counselling), throwing up another area to put more effort for improved result.

\section{Conclusion}

The study revealed that the 2015 ANC sero-prevalence for HIV in Kagini health center is $2.8 \%$ which is similar to the 2015 national ANC sero-prevalence of 3.0\%. Concerning ARV therapy initiation for the 40 sero-positive pregnant women, only 30 (75\%) of them were placed on ARV therapy which fell short of $100 \%$ expected. Furthermore, out of the 30 babies born to HIV positive mothers, only 10 (33.3\%) were placed on ARV therapy which again fell short of $100 \%$ expected. These figures fell grossly short of the expected performance and portend great danger to the goal of elimination of mother to child transmission of HIV.

\section{Recommendations}

1. The PMTCT program sponsors at this facility should retrain the HIV counsellors to position them for a robust post-test, pre-initiation and adherence counselling services at Kagini Health Center. This will ensure an increased ARV initiation and retention rate.

2. Furthermore, trained treatment support specialist (TSS) should be deployed to this facility for adequate treatment support and follow-up of clients and their babies.

3. Moreover, the facility health workers should also be supported in the area of creating awareness for early ANC booking within the host community and all the catchment area communities, this will go a long way to helping in early detection as well as early ARV initiation for improved PMTCT in addition to reducing maternal mortality.

4. Lastly, supportive supervision should be provided by the PMTCT program sponsors and the Local Government Health authority to periodically monitor the PMTCT 
activities to ensure the set targets are on course; this will eliminate laxity on the part of the health workers.

\section{Further research}

This present research did not review the area of HIV testing for the infants born to HIV positive women (infants exposed to HIV)

Therefore, future research to evaluate the Percentage of infants born to HIV positive women receiving a Virological test for HIV within 2 months of birth in addition to assessing the effectiveness of the PMTCT program by evaluating the prevalence of sero-negative children after 18 months of age.

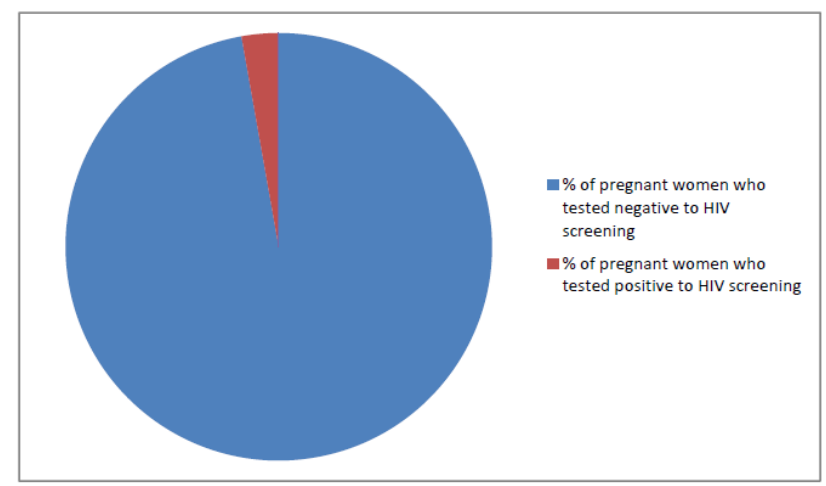

Figure 1

Pie chart representing the percentage of ANC Clients who are sero-positive and those who are seronegative to HIV screening test at Kagini Health Center in the year $2015 \mathrm{P}=40 / 1418=\mathbf{2 . 8 \%}$ prevalence.

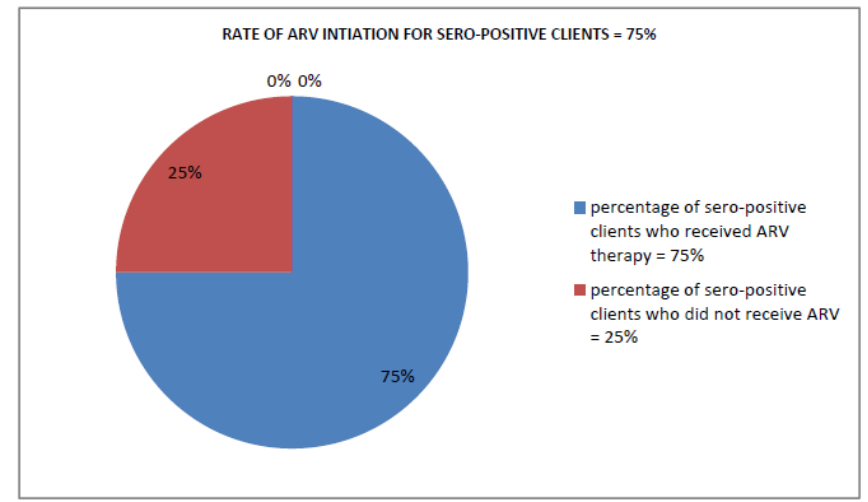

Figure 2

Pie chart representing the percentage of sero-positive pregnant clients who received ARV therapy $\mathrm{P}=$ 30/40 (75\%).

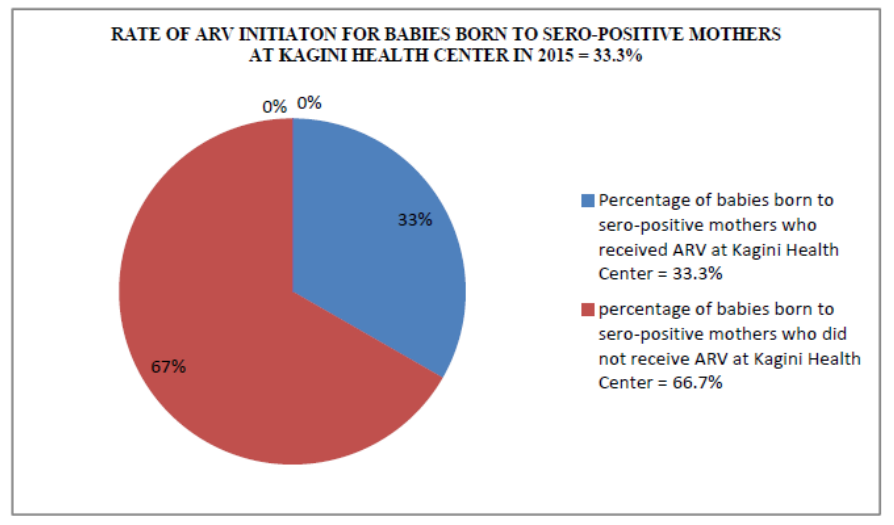

Figure 3 
Texila International Journal of Public Health

Volume 4, Issue 4, Dec 2016

Pie chart representing the percentage of Babies born to sero-positive mothers who received ARV therapy according to National guideline and those who do not at Kagini Health center in $2015 \mathrm{P}=$ $10 / 30(33.3 \%)$

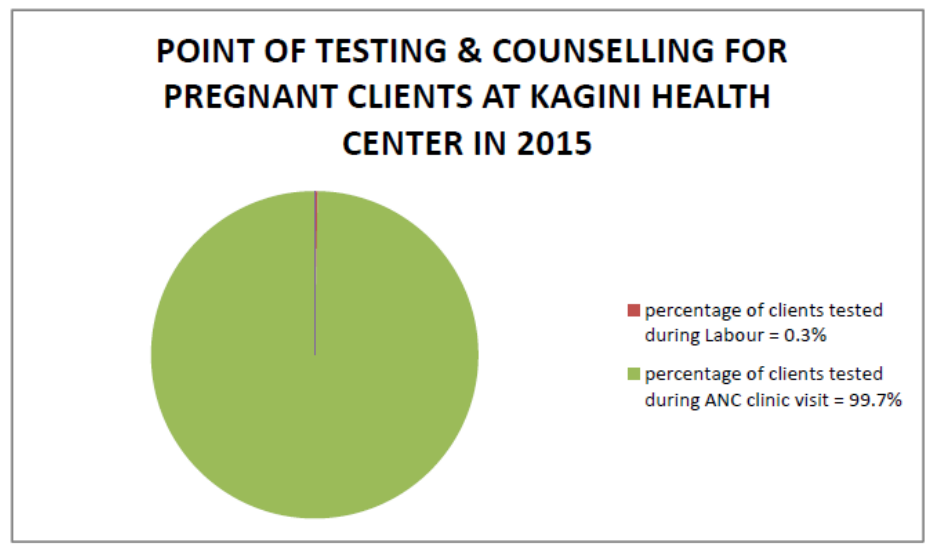

Figure 4

Pie chart representing the percentage of clients tested during ANC clinic and those whose initial presentation to the center was during Labour and thus tested while in labour at Kagini Health center in 2015. (ANC: 1414/1418 =99.7\%; Labour: 4/1418 = 0.3\%)

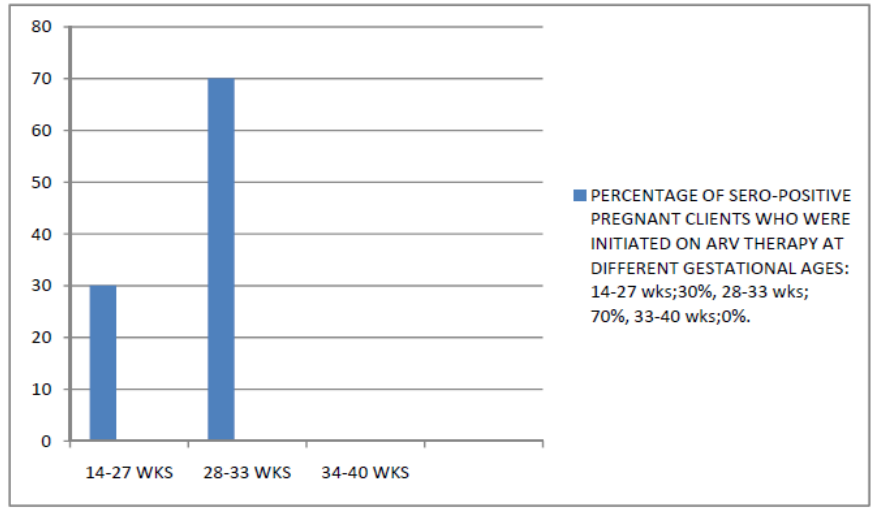

Figure 5

Bar chart showing the percentage of sero-positive pregnant clients who were initiated on ARV therapy and the gestational age at which it was initiated at Kagini Health Center in 2015 (14-27 weeks: 9/30 = 30\%; 28-33 weeks: $21 / 30=70 \%$; 34-40 weeks: 0/30 = 0\%)

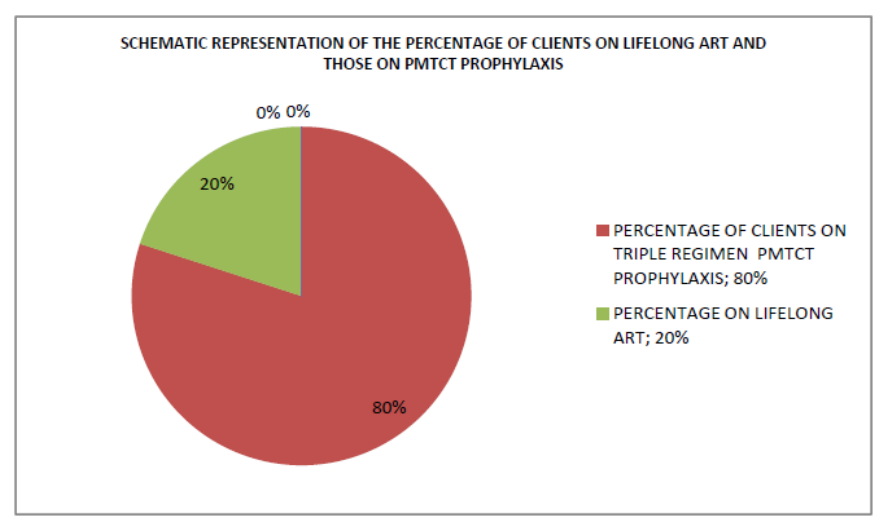

Figure 6

Pie chart showing the distribution of lifelong ART vs PMTCT triples regimen prophylaxis therapy among the sero-positive pregnant clients at Kagini Health Center in 2015. (Lifelong ART: 6/30 = 20\%; PMTCT prophylaxis: 24/30 = 80\%) 


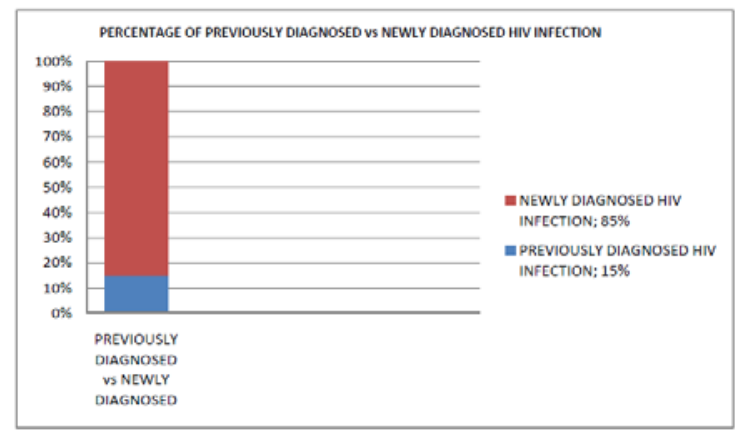

Figure 7

Column Chart showing the percentage of previously diagnosed HIV infection vs newly diagnosed HIV infection among pregnant women at Kagini Health Center in 2015. (Previously diagnosed: 6/40 = 15\%; newly diagnosed: $34 / 40=85 \%$ )

\section{References}

[1] AIDSINFO (2016). Education materials: HIV-1. Accessed from:

https://aidsinfo.nih.gov/education-materials/glossary/314/hiv-1

[2] FMOH of Nigeria, (2010). National Guideline for Prevention of Mother to Child Transmission (PMTCT) of HIV/AIDS. Pp 1

[3] FMOH, of Nigeria, HSS (2014). 2014 National HIV Sero-prevalence Sentinel Survey among Pregnant Women Attending Antenatal Clinics in Nigeria. pp 2.

[4] Ghana AIDS commission GAC (2013). Summary of the 2013 HIV sentinel survey report. Accessed from: http://ghanaids.gov.gh/gac1/aids_info.php

[5] IATT, (2015). Inter-agency Task Team on the Prevention and Treatment of HIV Infection in Pregnant Women, Mothers and Children. Monitoring \& Evaluation Framework for Antiretroviral Treatment for Pregnant and Breastfeeding Women Living with HIV and Their Infants. (IATT M\&E Option B+ Framework). CDC, WHO and UNICEF. New York. Accessed from: http://www.emtctiatt.org/wp-content/uploads/2015/05/IATT-Framework-May-2015.pdf

[6] Kenya AIDS Response Progress Report (2014). Progress towards zero. Accessed

from http://www.unaids.org/sites/default/files/country/documents/KEN_narrative_report_2014.pdf

[7] Kiptoo M., Mpoke S., Ng'ang'a Z., Mueke J., Okoth F., Songok E. (2009). Survey on prevalence and risk factors on HIV-1 among pregnant women in North-Rift, Kenya: a hospital based crosssectional study conducted between 2005 and 2006, 9: pp10. doi: 10.1186/1472-698X-9-10.

[8] National Agency for the Control of AIDS (NACA) (2014). Federal republic of Nigeria Global AIDS Response. Country Progress Report, Nigeria GARPR 2014. p.13 Accessed from: http://www.unaids.org/sites/default/files/country/documents/NGA_narrative_report_2014.pdf [9] Okerentugba P.O., Uchendu S.C., Okonko I.O. (2015). Prevalence of HIV among Pregnant Women in Rumubiakani, Port Harcourt. Nigeria. Public Health Research. 5(2): 5865.doi:10.5923/j.phr.20150502.03

[10] Sagay A.S., Kapiga S.H., Imade G.E., Sankale J.L., Idoko J., Kanki P. (2005). HIV Infection among pregnant women in Nigeria. International journal of Gynecology and Obstetrics, 90: (1). Pp 6167. Doi 10.1016/j.jigo.2005.30.030

[11] Thailand National AIDS Committee (NAC). (2015). Thailand AIDS response progress report, 2015. Accessed from http://www.unaids.org/sites/default/files/country/documents/THA_narrative_report_2015.pdf

[12] United Nations. (2015). Sustainable Development Goals (SDGs). Accessed from:

http://www.un.org/sustainabledevelopment/sustainable-development-goals/

[13] $\mathrm{WHO}_{1}$ (2016). HIV/AIDS, online Q \& A. (updated July, 2016). Accessed from: http://www.who.int/features/qa/71/en/

[14] $\mathrm{WHO}_{2}$ (2016). HIV/AIDS, Mother-to-child transmission of HIV (updated June, 2016). Accessed from: http://www.who.int/hiv/topics/mtct/en/

[15] $\mathrm{WHO}_{3}$ (2016). Thailand is first country in Asia to eliminate mother-to-child transmission of HIV and syphilis. Accessed from: http://www.searo.who.int/mediacentre/releases/2016/1627/en/index.html 
Texila International Journal of Public Health

Volume 4, Issue 4, Dec 2016

[16] $\mathrm{WHO}_{4}$ (2016). WHO validates elimination of mother-to-child transmission of HIV and syphilis in Armenia, Belarus and the Republic of Moldova. (Updated June, 2016). Accessed from:

http://www.euro.who.int/en/media-centre/sections/press-releases/2016/06/who-validates-eliminationof-mother-to-child-transmission-of-hiv-and-syphilis-in-armenia,-belarus-and-the-republic-of-moldova. [17] $\mathrm{WHO}_{5}$ (2016). Consolidated guidelines on the use of antiretroviral drugs for treating and preventing HIV infection. Summary of Recommendations. Accessed from:

http://www.who.int/hiv/pub/arv/summary-recommendations.pdf 